Delft University of Technology

\title{
A 30 ppm $<80$ nJ Ring-Down-Based Readout Circuit for Resonant Sensors
}

Jiang, H; Chang, ZY; Pertijs, MAP

DOI

10.1109/JSSC.2015.2470552

Publication date

2015

Document Version

Accepted author manuscript

Published in

IEEE Journal of Solid State Circuits

\section{Citation (APA)}

Jiang, H., Chang, ZY., \& Pertijs, MAP. (2015). A 30 ppm < 80 nJ Ring-Down-Based Readout Circuit for Resonant Sensors. IEEE Journal of Solid State Circuits, 51(1), 187-195.

https://doi.org/10.1109/JSSC.2015.2470552

\section{Important note}

To cite this publication, please use the final published version (if applicable).

Please check the document version above.

Other than for strictly personal use, it is not permitted to download, forward or distribute the text or part of it, without the consent of the author(s) and/or copyright holder(s), unless the work is under an open content license such as Creative Commons.

\section{Takedown policy}

Please contact us and provide details if you believe this document breaches copyrights.

We will remove access to the work immediately and investigate your claim. 


\title{
A 30ppm $<$ 80nJ Ring-Down-Based Readout Circuit for Resonant Sensors
}

\author{
H. Jiang, Z.Y. Chang and M.A.P. Pertijs \\ Electronic Instrumentation Laboratory, Delft University of Technology \\ Delft, The Netherlands
}

\begin{abstract}
This paper presents an energy-efficient readout circuit for micro-machined resonant sensors. It operates by briefly exciting the sensor at a frequency close to its resonance frequency, after which resonance frequency and quality factor are determined from a single ring-down transient. The circuit employs an inverter-based trans-impedance amplifier to sense the ring-down current, with a programmable feedback network to enable the readout of different resonant sensors. An inverter-based comparator with dynamically-adjusted threshold levels tracks the ring-down envelope to measure quality factor, and detects zero crossings to measure resonance frequency. The excitation frequency is dynamically adjusted to accommodate large resonance frequency shifts. Experimental results obtained with a prototype fabricated in $0.35 \mu \mathrm{m}$ standard CMOS technology and three different SiN resonators are in good agreement with conventional impedance analysis. The prototype achieves a frequency resolution better than $30 \mathrm{ppm}$ while consuming less than $80 \mathrm{~nJ} / \mathrm{meas}$ from a $1.8 \mathrm{~V}$ supply, which is $7.8 \times$ less than the state-of-the-art.
\end{abstract}

Index Terms-MEMS resonant sensors readout, energy efficiency, ring-down measurement, frequency tracking technique, inverter-based front-end. 


\section{INTRODUCTION}

Resonant sensors are widely applied for various physical measurements, such as pressure sensing, mass detection, oil-quality measurement and gas sensing. Generally, in these systems, a change in the physical quantity of interest leads to a shift in the resonance frequency $f_{\text {res }}$ of a mechanical resonator. By equipping the resonator with an appropriate capacitive or piezoelectric transducer, $f_{\text {res }}$ can be detected and digitized by a readout circuit. Micro- or nano-mechanical resonators are an attractive choice for energy-constrained applications, because they can be operated at very low energy levels. For instance, piezoelectrically actuated $\mathrm{SiN}$ resonators have been reported that can detect ppm-level concentrations of volatile organic compounds at power levels below 100nW [1]. For such resonators, an energyefficient readout circuit is required, since the overall energy consumption is readily dominated by the circuit.

Resonant sensors are typically read out using an oscillator-based circuit [2 - 4]. This is illustrated in Fig. 1a, in which the resonator is modeled by a resonant circuit (consisting of $R_{m}$, $C_{m}$ and $L_{m}$ ), representing the mechanical resonance, in parallel with a feed-through capacitance $C_{p}$. A sustaining amplifier, typically a trans-impedance amplifier, senses the current produced by the resonator and feeds back a drive signal that ensures $360^{\circ}$ phase shift around the loop, so that oscillation is maintained at $f_{\text {res. }}$. A comparator translates the drive signal to logic levels, after which a counter digitizes its frequency relative to a reference clock.

Oscillator-based readout circuits tend to be relatively power hungry [2, 3]. This is mainly because the bandwidth of the sustaining amplifier must be substantially higher than $f_{\text {res }}$, in order to prevent the phase shift that it introduces in the feedback loop from moving the oscillation frequency significantly away from $f_{\text {res }}$. Moreover, it is difficult to design oscillatorbased readout circuits for piezoelectric resonators with relatively large parasitic feed-through 
capacitance, because this capacitance, rather than the mechanical resonance, will determine the phase shift at $f_{\text {res. }}$. In this case, the effect of $C_{p}$ needs to be compensated for, for instance using a bridge configuration with a replica device driven out-of-phase [2], which will increase area and power consumption. Finally, oscillator-based readout circuits do not readily provide information about the resonator's quality factor $Q$, while this can also provide valuable information, for example, about surface binding or pressure changes, which relate to damping and energy-loss effects [5].

As an alternative, ring-down based readout, illustrated in Fig. 1b, can be more energyefficient, is capable of detecting both $f_{\text {res }}$ and $Q$, and can operate in the presence of large parasitic capacitance $[6-10]$. The basic idea of ring-down measurement is to operate the resonator as a tuning fork: excite it and let it ring down at $f_{\text {res. }}$ To excite the resonator, an excitation source drives the transducer in order to add sufficient energy to the desired resonant mode. When it is turned off, the sensor dissipates the energy while vibrating at $f_{\text {res. }}$. This produces an exponentially-decaying current through the piezoelectric transducer, which is converted to a voltage by an I-V converter. From the zero crossings of this voltage, $f_{\text {res }}$ can be extracted, while its envelope contains information about $Q$. Integrated readout circuits based on this technique have been reported [8,9], and consume significantly less energy than oscillator-based readout circuits for similar sensors $[2,3]$. These designs, however, require three or more ring-downs to measure both $f_{\text {res }}$ and $Q$, while this information can, in principle, be obtained from a single ring-down, and thus at significantly lower energy consumption.

This paper describes the architecture, circuit realization and experimental characterization of an energy-efficient ring-down-based readout circuit for resonant sensors [10]. Unlike previous designs, it combines $f_{\text {res }}$ and $Q$ measurements in a single ring-down. It employs a comparator with a dynamically-adjusted threshold and a current-efficient inverter- 
based front-end. A prototype realized in $0.35 \mu \mathrm{m}$ standard CMOS technology achieves $1.6 \times$ better resolution at $7.8 \times$ lower energy consumption than prior ring-down-based designs.

This paper is organized as follows. In Section II, the architecture and the operating principle of the proposed ring-down-based readout circuit are described. Sections III and IV discuss the implementation details of the circuit and present a noise analysis, respectively. Section V describes the measurement results. The paper concludes in Section VI.

\section{BASIC OPERATING PRINCIPLE}

\section{A. Target Sensors}

The readout circuit described in this paper has been optimized for use with silicon-nitride (SiN) clamped-clamped beam resonators with integrated piezoelectric (aluminum nitride) transducers [1]. Their length ranges from $100 \mu \mathrm{m}$ to $1000 \mu \mathrm{m}$, their width is between $20 \mu \mathrm{m}$ and $100 \mu \mathrm{m}$, and the SiN thickness is about $500 \mathrm{~nm}$. A typical impedance characteristic around $f_{\text {res }}$, measured using a bench-top impedance analyzer, is shown in Fig. 2. At frequencies close to the resonance frequency of approximately $536 \mathrm{kHz}$, the resonator can be modeled as an $R L C$ tank (motional resistance $R_{m}$, inductance $L_{m}$ and capacitance $C_{m}$ ) in parallel with a capacitor $C_{p}$ that models the capacitance of the piezoelectric transducer and any parasitic capacitances. The readout circuit aims to measure $f_{\text {res }}$ and $Q$ of the $R L C$ branch, where

$$
f_{\text {res }}=\frac{1}{\sqrt{L_{m} C_{m}}}, \quad Q=\frac{1}{R_{m}} \sqrt{\frac{L_{m}}{C_{m}}}
$$

The relative resolution for the measurement of $f_{\text {res }}$ targeted in this work is $30 \mathrm{ppm}$. The impact of $C_{p}$ on the impedance is evident from the fact that the resonator exhibits only a small phase shift around resonance. This makes it difficult to implement an oscillator-based readout circuit for this type of resonators, unless the effect of $C_{p}$ is compensated for [2, 3]. 
B. Readout Architecture

The basic architecture of the ring-down-based readout circuit is shown in Fig. 3. It consists of a switched excitation source, a trans-impedance amplifier (TIA), a comparator and a digital counter. There are two operating phases in the ring-down measurement: an excitation phase $\phi_{\text {exc }}$ and a ring-down phase $\phi_{\text {ring. }}$. During $\phi_{\text {exc }}$, the sensor is driven by the excitation source $V_{\text {exc }}$, which has a frequency close to $f_{\text {res }}$, and starts oscillating at the driving frequency. Then, during $\phi_{\text {ring, }}$, the driving source is turned off and the sensor is connected to the readout circuit. Due to the energy that has been accumulated during the excitation phase, the sensor starts to produce an oscillating current at $f_{\text {res }}$, with an exponentially-decaying envelope [6]. This ringdown current is turned into a voltage $V_{\text {ring }}$ by the TIA. The comparator detects the zerocrossings or threshold-crossings of this voltage, from which $f_{\text {res }}$ and $Q$ are derived.

\section{Resonance Frequency Measurement}

Zero-crossing detection and frequency counting are used to measure $f_{\text {res }}$, as illustrated in Fig. 4a. To detect the zero-crossings of $V_{\text {ring }}$, the threshold voltage of the comparator is set to $V_{t h 0}$, which corresponds to the average of $V_{\text {ring. }}$. The resonance frequency is then extracted by counting the number of cycles $M$ of a reference clock, with period $T_{0}$, during a fixed number of $N_{\text {total }}$ zero-crossings of the ring-down signal:

$$
f_{\text {res }}=\frac{N_{\text {total }}}{M T_{0}}
$$

D. Quality Factor Measurement

The envelope of the ring-down signal contains information about the quality factor $Q$ of the resonant sensor. To digitize $Q$, threshold-crossing detection can be applied [7]: as shown in 
Fig. 4a, in successive ring-downs, the comparator is operated at two different threshold levels $V_{t h 1}$ and $V_{t h 2}$, and detects the time instants at which the envelope drops below these levels, which are quantized by counting the corresponding number of threshold crossings $N_{1}$ and $N_{2}$. An approximation of $Q$ can then be obtained from the formula [7]

$$
Q=\frac{\pi\left(N_{2}-N_{1}\right)}{\ln \left(\frac{V_{t h 1}-V_{t h 0}}{V_{t h 2}-V_{t h 0}}\right)}
$$

\section{E. Proposed Combined Measurement}

In previous integrated implementations of the ring-down technique $[8,9]$, multiple successive excitation and ring-down phases were applied to determine $f_{\text {res }}$ and $Q$. The readout circuit presented in this paper, in contrast, determines $f_{\text {res }}$ and $Q$ from one ring-down signal, which reduces measurement time and energy consumption.

As illustrated in Fig. 4b, a single comparator with a dynamically-adjusted threshold is used. The threshold level starts at $V_{t h}$, which corresponds to the steady-state level of ringdown signal. After a few cycles, it is switched to a higher threshold $V_{t h 1}$ and then switched back in steps to $V_{t h 0}$. These threshold-switching points happen when the envelope of the ringdown signal drops below the relevant threshold level. Then, the number of the threshold crossings $\left(N_{1}, N_{2}\right)$ can be counted, from which $Q$ can be calculated using equation (3).

The counting continues until a pre-defined number of $N_{\text {total }}$ ring-down cycles has been reached. Thus, as before, $f_{\text {res }}$ can be obtained using equation (2), by counting the number of references-clock cycles $M$ within these $N_{\text {total }}$ cycles.

A time-out mechanism is used to decide the threshold-switching points. A time-out detector counts the number of reference-clock cycles that have passed since the last level crossing, as illustrated in Fig. 5. If this number exceeds a time-out level, the envelope is assumed to have dropped below the threshold. This time-out level is set roughly equal to one 
cycle of the ring-down signal by employing the same timer to measure the duration of the first cycle. After a time-out, the threshold is switched to $V_{t h 0}$ first, to ensure that a next level crossing can be found by the comparator. Once found, the threshold is switched to the next threshold level. The frequency of the reference clock is about 40 times higher than $f_{\text {res }}$ to achieve a sufficiently accurate time-out detection.

\section{F. Excitation Signal and Frequency Tracking}

Ring-down measurement requires that the resonator absorbs enough energy in its resonance mode during the excitation phase. In principle, the resonator can be excited by an impulse. However, for the resonators used in this work, the required voltage amplitude would be beyond the capability of standard CMOS technology and would drive the resonator into its nonlinear region. Moreover, if the resonator has multiple dominant resonance modes, impulse excitation will cause the resonator to vibrate at all modes simultaneously, complicating accurate ring-down measurement. Therefore, instead, we use periodic excitation at a fixed frequency close to the target resonance mode. Thus, an excitation signal with smaller voltage amplitude can be used to excite resonator sensor, and excitation of undesired resonance modes can be prevented. For implementation simplicity, square-wave excitation is used.

The initial amplitude of the ring-down signal depends on the frequency of the excitation signal. By sweeping the frequency of the excitation signal near the resonance, a maximum ring-down current can be found when the driving frequency is exactly equal to $f_{\text {res }}$, at which the impedance of the resonator is minimum and equals value $R_{m}$. The initial amplitude of the ring-down current $I_{R L C}$ decreases as the difference between excitation frequency and the resonance frequency $\Delta f$ increases, as expressed by the following equation: 


$$
I_{R L C}=\frac{V_{\text {exc }}}{R_{m} \cdot \sqrt{1+\left(2 \frac{f_{\text {res }}-f_{\text {exc }}}{f_{\text {res }}} Q\right)^{2}}}
$$

where $V_{\text {exc }}$ and $f_{\text {exc }}$ are the amplitude and frequency of a sinusoidal excitation signal, respectively. (For square-wave excitation, this corresponds to the first harmonic of the excitation signal.) Hence, in order to get a sufficiently large ring-down current, the excitation frequency should be close enough to $f_{\text {res }}$, relative to the bandwidth $f_{\text {res }} / Q$, which is about $1.5 \mathrm{kHz}$ for our sensor.

Because $f_{\text {res }}$ shifts in response to changes in the physical quantity being measured, a fixed excitation frequency can typically not be used. Instead, after a ring-down measurement, the excitation frequency is updated based on the measured $f_{\text {res }}$. For a slowly-changing $f_{\text {res }}$, this will keep the excitation frequency within the bandwidth of the resonator. After startup, or in case of larger abrupt changes in $f_{r e s}$, the excitation frequency is swept across the frequency range of interest in steps small enough to allow the resonance to be found. With this approach, typically only a single excitation will be needed per measurement.

\section{CIRCUIT REALIZATION}

Fig. 6 shows a block diagram of the proposed readout circuit, which consists of an inverterbased TIA with reconfigurable feedback network, a comparator, a sensor driver, a period time-out detector and a capacitive DAC (CDAC). Control signals and the excitation clock are generated off-chip using an FPGA for flexibility.

\section{A. TIA}

The TIA is used to convert the ring-down current to a ring-down voltage for the comparator. It should have a small enough input impedance to ensure that most of the motional current 
flows into the TIA instead of into the parasitic capacitor $C_{p}$; a high enough trans-impedance to produce an output voltage with sufficient initial amplitude; and good enough noise performance for the targeted resolution, as will be discussed in Section IV-B. These requirements lead to a minimum trans-conductance for the operational transconductance amplifier (OTA) of the TIA.

To optimize the energy efficiency, an inverter-based OTA is used, as shown in Fig. 7 [11]. An auto-zeroing phase is adopted to set the bias point of the OTA's transistors using a floating current source. The difference between the gate voltages of these transistor and the input bias voltage is stored on capacitors $C_{A Z 1}$ and $C_{A Z 2}$. Thus, the sensitivity of the bias current and the DC output voltage to process, supply-voltage and temperature variations is significantly less than in a conventional inverter [11]. The input transistors are biased in weak-inversion to optimize current efficiency. The output swing is limited to $1 \mathrm{~V}$ to prevent distortion of the ring-down envelope from causing significant errors in the $Q$ measurement.

The TIA's feedback network has been made reconfigurable to allow the circuit to measure different resonant sensors. The gain value of TIA can be programmed from $160 \mathrm{k} \Omega$ to $5.12 \mathrm{M} \Omega$. The TIA consumes about $12 \mu \mathrm{A}$.

\section{B. Comparator}

Propagation delay of the comparator results in an error in $f_{\text {res }}$, since the input signal of the comparator has an exponentially decaying envelope, and the propagation delay is typically amplitude-dependent. Hence the delay at the start of the ring-down will be smaller than that at the end, and will not cancel out in the time measurement. Therefore, we design the comparator to have a delay that is sufficiently small compared to the resonance period. To do so in an energy-efficient way, an inverter-based auto-zeroing comparator is utilized. 
Fig. 8 shows the circuit diagram of the comparator, which consists of two inverter stages. The input stage is dynamically biased by means of an auto-zeroing scheme similar to that of OTA in the TIA (but without cascode transistors). During the auto-zeroing phase, the two input transistors $M_{n 1}$ and $M_{p 1}$ are diode-connected and biased at the same bias current by a floating current source, while their gate-source voltages, including offset and $1 / f$ noise, are stored on the capacitors $C_{A Z C 1}$ and $C_{A Z C 2}$. The floating current source is kept in the circuit during the comparison phase and assists in class-AB biasing of the output stage, thus reducing its static current consumption while maintaining a high slew rate. The comparator consumes about $8 \mu \mathrm{A}$ supply current during operating. The difference in propagation delay between a $20 \mathrm{mV}$ peak-to-peak input voltage and a $400 \mathrm{mV}$ peak-to-peak input voltage is below $18 \mathrm{~ns}$.

\section{On-chip Sensor Driver}

As shown in Fig. 6, the square-wave excitation signal is generated using an on-chip driver, which is clocked by an off-chip excitation clock. The circuit diagram of the driver is shown in Fig. 9. When $\phi_{\mathrm{EX}}$ is high, $V_{e x c}$ is pulled to ground by $M_{n 8}$. When $\phi_{\mathrm{EX}}$, is low, the output $V_{e x c}$ is pulled up by source follower $M_{n 9}$. The input of this source follower is derived from a reference voltage $V_{\text {ref }}$ using a diode-connected transistor $M_{n 6}$. Thus, the amplitude of $V_{\text {exc }}$ is defined by $V_{r e f}$. Transistors $M_{p 3}$ and $M_{n 7}$ power down the source follower and its biasing when $\phi_{\mathrm{EX}}$ is high. When active, the sensor driver consumes an average supply current of $1 \mu \mathrm{A}$.

\section{Implementation and Timing Details}

As shown in Fig. 10, several auto-zeroing steps are applied to correct for the offset of the front-end and other errors in every ring-down measurement, so as to maximize the number of zero-crossings that can be detected. 
Before the excitation phase, the OTA and comparator are auto-zeroed, as described above. In Fig. 10, single offset-storage capacitors are shown at their inputs for simplicity, but the actual implementation involves pairs of capacitors, as shown in Fig. 7 and 8. During phase $\phi_{\mathrm{AZ}}$, both the OTA and the comparator are configured in unity-gain feedback, so that their input offset is stored on capacitors $\mathrm{C}_{\mathrm{AZ}}$ and $\mathrm{C}_{\mathrm{AZC}}$, respectively. Then, during $\phi_{\mathrm{AZC}}$, the comparator is kept in unity-gain feedback, while the OTA is connected to its feedback network and the resonator. By doing this, any residual output offset of the TIA will be stored on capacitor $C_{c}$, which capacitively couples the TIA's output to the input of the comparator.

The binary-weighted capacitors of the 3-bit CDAC are also capacitively coupled to the comparator's input and are driven using logic signals $\mathrm{D}_{1,2,3}$. During auto-zeroing, these signals are zero. During the ring-down phase, the CDAC's inputs are switched, changing the voltage on $C_{c}$ via charge-redistribution, and thus changing the comparator's threshold as desired.

After auto-zeroing, the sensor is excited during phase $\phi_{\text {exc. }}$ Then, the feed-through parasitic capacitor $C_{p}$ is discharged during phase $\phi_{\mathrm{Cp}}$, in order to prevent the charge on $C_{p}$ from shifting the output of the TIA during the ring-down. As shown Fig. 11, when $C_{p}$ is large compared to the sensor's impedance, any charge that has been left on $C_{p}$ at the end of excitation phase will cause an undesired transient at the output of TIA, which will cause errors in the $Q$ measurement. This is solved by introducing a short phase $\phi_{\mathrm{Cp}}$ to discharge $C_{p}$ after the excitation phase to make the time for recovery shorter. In order to realize this, the TIA is held in unity-gain during $\phi_{\mathrm{cp}}$.

\section{NOISE ANALYSIS}

The resolution of the resonance frequency measurement is determined by two effects: quantization errors and timing jitter associated with the thermal noise sources in the circuit. 


\section{A. Quantization Errors}

In order to quantize the duration of the $N_{\text {total }}$ ring-down cycles, a digital counter, realized on FPGA, counts the number of cycles $M$ of a reference clock. This is associated with two quantization errors, which are uncorrelated, at the first cycle and the last cycle, leading to an RMS quantization error $\left(\sigma_{q}\right)$ of

$$
\sigma_{q}\left(\frac{\Delta f}{f}\right)=\frac{f_{\text {res }}}{\sqrt{6} N_{\text {total }} f_{c l k}}
$$

where $f_{c l k}$ is the reference clock frequency. To arrive at a resolution better than $30 \mathrm{ppm}$, with $400 \mathrm{kHz}<f_{\text {res }}<600 \mathrm{kHz}$ and $300<N_{\text {total }}<600, f_{\text {clk }}$ should be at least $25 \mathrm{MHz}$.

\section{B. Timing Jitter}

There are two dominant thermal-noise sources in the ring-down-based readout circuit: noise of the TIA and noise of the comparator. The combined noise at the input of comparator can be estimated by adding the TIA's output noise and the equivalent input noise of the comparator. This combined noise will result in timing jitter in the zero-crossing detection. The expression for the RMS timing jitter $\Delta t$ in terms of the initial ring-down signal amplitude $A_{0}$, the combined rms voltage noise $v_{n}$ and the ratio of $N_{\text {total }}$ and $Q$ is obtained as

$$
\Delta t=\frac{v_{n}}{2 \pi f_{\text {res }} A_{0} e^{-\frac{\pi N_{\text {total }}}{Q}}}
$$

in which the denominator represents the slope of at the zero-crossings of the ring-down signal at the end of the measurement. By assuming $N_{\text {total }} \approx Q$, the timing jitter simplifies to

$$
\Delta t=\frac{v_{n}}{2 \pi f_{\text {res }} A_{0} e^{-\pi}}
$$

The uncertainty in the resonance-frequency measurement as a result of this timing jitter can be expressed as 


$$
\sigma_{n}\left(\frac{\Delta f}{f}\right)=\frac{\Delta t \cdot f_{\text {res }}}{N_{\text {total }}}=\frac{v_{n}}{2 \pi A_{0} N_{\text {total }} e^{-\pi}}
$$

Using this equation, the targeted resolution can be translated into a requirement on the signalto-noise ratio $A_{0} / v_{n}$ at the start of the ring-down, which for $N_{\text {total }}=Q=300$ is about 430 and determines the current levels in the inverter-based OTA and comparator.

\section{MEASUREMENT RESULTS}

The readout circuit has been realized in $0.35 \mu \mathrm{m}$ standard CMOS technology and tested with three different resonators with $f_{\text {res }}=469 \mathrm{kHz}, 537 \mathrm{kHz}, 592 \mathrm{kHz}$ and $\mathrm{Q}=521,386,376$ (under ambient conditions). The TIA's reconfigurable feedback network was used to adapt its gain to these different resonators. Fig. 12 shows a micrograph of the chip, with an active area of $0.24 \mathrm{~mm}^{2}$, and of the three resonant sensors. The readout operates from supply voltages from $1.8 \mathrm{~V}$ to $3.3 \mathrm{~V}$. The experimental results reported here were obtained at a supply voltage of $1.8 \mathrm{~V}$, at which the current consumption is $34 \mu \mathrm{A}$, including the current consumption of the on-chip driver that provides energy to the resonator and of the period time-out detector.

The resolution of the measured $f_{\text {res }}$ as a function of the number of ring-down cycles $N_{\text {total }}$ is shown in Fig. 13. The resolution at a particular value $N_{\text {total }}$ is calculated as the standard deviation of 1000 successive measurements. When $N_{\text {total }}$ is smaller than 200 , the resolution is limited by the quantization noise associated with the $25 \mathrm{MHz}$ reference clock of the counter, in good agreement with equation (5). For larger values of $N_{\text {total }}$, jitter due to the thermal noise of the TIA and the comparator limits the resolution. For a measurement time of $1.3 \mathrm{~ms}$, including all phases of the ring-down measurement, an optimum better than $30 \mathrm{ppm}$ can be reached for all three resonators when $N_{\text {total }}$ is roughly equal to $Q$. The resolution of $Q$ is limited by quantization noise and is better than $1 \%$. 
To demonstrate the combined measurement of $f_{\text {res }}$ and $Q$, the three resonant sensors have been exposed to different pressure levels in a vacuum chamber. Pressure is swept from 200 mbar to 1000 mbar, which leads to significant changes in $Q$ due to changing air damping, and a smaller variation in $f_{\text {res }}$, as shown in Fig. 14. The results obtained using our readout circuit are in good in agreement with results from a bench-top impedance analyzer (Agilent 4294A). The mismatch in $f_{\text {res }}$ between the two data sets is smaller than $200 \mathrm{~Hz}$ and that in $Q$ is smaller than 15 . The $Q$ values obtained using our readout circuit are systematically slightly lower than those obtained with the impedance analyzer. This is likely due to the finite input resistance of the TIA, which slightly increases the losses in the resonator.

In order to illustrate the frequency tracking technique, one resonant sensor and a reference PT100 thermometer have been exposed in a climate chamber to temperature changes, since these will cause a significant variation of $f_{\text {res }}$. Temperature is varied step-wise between $10^{\circ} \mathrm{C}$ and $40^{\circ} \mathrm{C}$ while the humidity level is kept constant. As shown in Fig. 15, a $30^{\circ} \mathrm{C}$ temperature variation causes a frequency shift of about $8 \mathrm{kHz}$, which far exceeds the bandwidth of the resonator of $1.37 \mathrm{kHz}$ and can therefore not be measured using a fixed excitation frequency. The ring-down measurement circuit, employing the frequency-tracking technique, nevertheless tracks this large shift and maintains a good resolution (31ppm).

For 9 samples of the readout chip, the measured sample-to-sample variation in $f_{\text {res }}$ and $Q$ (for the same resonator under constant ambient conditions), is less than $\pm 90 \mathrm{~Hz}$ and \pm 2.5 , respectively. The measured shift of $f_{\text {res }}$ and $Q$ due to power supply variation in the range from $1.8 \mathrm{~V}$ to $3.3 \mathrm{~V}$ is below $100 \mathrm{~Hz}$ and 3, respectively. (In these measurements, averaging was applied to properly distinguish between systematic effects and noise).

In Table I, the performance of the readout circuit is summarized and compared with several previously published readout circuits for similar resonant sensors. Oscillator-based readout circuits $[2,3]$ achieve similar or better resolution, however, at much higher power 
consumption and much longer measurement time, without providing information about $Q$. Compared with earlier ring-down based circuits [9], this readout circuit consumes $1.7 \times$ less power and provides a $1.6 \times$ better resolution in a $5 \times$ shorter measurement time .

\section{CONCLUSION}

Ring-down-based readout of resonant sensors is a promising readout approach for use in energy-constrained applications. Compared with traditional oscillator-based readout, it can determine $f_{\text {res }}$ at much lower energy consumption. Moreover, it is insensitive to the parasitic feedthrough capacitance and allows simultaneous $Q$ measurement. The proposed readout circuit, optimized for $\mathrm{SiN}$ micro-machined resonant sensors, is implemented in $0.35 \mu \mathrm{m}$ standard CMOS technology and has an active area of $0.24 \mathrm{~mm}^{2}$. It employs a dynamicallyswitching inverter-based level-crossing detector to determine $f_{\text {res }}$ and $Q$ from a single ringdown transient signal. The frequency tracking technique, rather than the use of a fixed excitation frequency, allows the detection of large shifts in $f_{r e s}$. A programmable feedback network is applied to enable the readout of different resonant sensors. The results obtained with three different resonant sensors are in good agreement with conventional impedance analysis. The circuit achieves a frequency resolution of $30 \mathrm{ppm}$ while consuming less than $80 \mathrm{~nJ} /$ meas from a $1.8 \mathrm{~V}$ supply, which is $7.8 \times$ less than the state-of-the-art.

\section{ACKNOWLEDGMENT}

The authors thank imec / Holst Center for providing prototype resonators, and Mr. Lukasz Pakula for his assistance in the pressure measurements. 


\section{REFERENCES}

[1] D. M. Karabacak, S. H. Brongersma and M. Crego-Calama, "Enhanced sensitivity volatile detection with low power integrated micromechanical resonators," Lab on a Chip, no. 10, pp. 1976-1982, May 2010.

[2] V. Petrescu, J. Pettine, D. M. Karabacak, M. Vandecasteele, M. Crego-Calama, C. Van Hoof, "Power-Efficient Readout Circuit for Miniaturized Electronic Nose," ISSCC Dig. Tech. Papers, pp. 318-319, Feb. 2012.

[3] J. Pettine, V. Petrescu, D. M. Karabacak, M. Vandecasteele, M. Crego-Calama, and C. Van Hoof, "Power-Efficient Oscillator-Based Readout Circuit for Multichannel Resonant Volatile Sensors," IEEE Trans. Biomedical Circuits and Systems, vol. 6, no. 6, pp. 542-551, Dec. 2012.

[4] Y.-W. Lin , S. Lee, S.-S. Li , Y. Xie , Z. Ren and C. T. C. Nguyen, "Series-Resonant VHF Micromechanical Resonator Reference Oscillators," IEEE J. of Solid-State Circuits, vol. 39, no. 12, pp. 2477-2491, Dec. 2004

[5] D. M. Karabacak, V. Yakhot, and K. L. Ekinci, "High-frequency nanofluidics: An experimental study using nanomechanical resonators," Physical Review Letters, vol. 98, no. 25, June 2007.

[6] K. Zeng, K. G. Ong, C. Mungle, and C. A. Grimes, "Time domain characterization of oscillating sensors: Application of frequency counting to resonance frequency determination," Review of Scientific Instruments, vol. 73, no. 12, pp. 4375-4380, Dec. 2002.

[7] K. Zeng and C. A. Grimes, "Threshold-crossing counting technique for damping factor determination of resonator sensors," Review of Scientific Instruments, vol. 75, no. 12, pp. 5257-5261, Dec. 2004. 
[8] Z. Zeng, M. A. P. Pertijs and D. M. Karabacak, "An Energy-Efficient Readout Circuit for Resonant Sensors Based on Ring-Down Measurement," Review of Scientific Instruments, vol. 84, no. 2, Feb. 2013.

[9] Y. Yan, Z. Zeng, C. Chen, H, Jiang, Z. Y. Chang, D. M. Karabacak and M. A. P. Pertijs, “An Energy-Efficient Reconfigurable Readout Circuit for Resonant Sensors Based on Ringdown Measurement," Proc. IEEE Sensors, in press, Nov. 2014.

[10] H. Jiang, Z. Y. Chang and M. A. P. Pertijs, “A 30ppm <80nJ ring-down-based readout circuit for resonant sensors," ISSCC Dig. Tech. Papers, pp. 482-483, Feb. 2015.

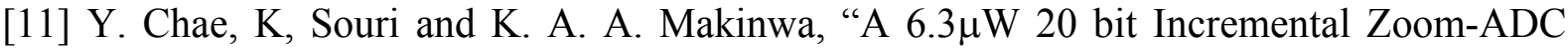
with 6ppm INL and $1 \mu \mathrm{V}$ Offset," IEEE J. of Solid-State Circuits, vol. 48, no. 12, pp. 30193027, Dec. 2013. 


\section{Figure captions:}

Fig. 1: Block diagram of (a) an oscillator-based readout circuit; (b) the proposed ring-downbased readout circuit.

Fig. 2: Measured typical impedance characteristic of the piezo-electrically actuated SiN resonators targeted in this work.

Fig. 3: Basic architecture of the ring-down measurement circuit.

Fig. 4: Timing diagram of $f_{\text {res }}$ and $Q$ measurements (a) using multiple successive ring-downs;

(b) combined in a single ring-down.

Fig. 5: Dynamic threshold adjustment for the combined $f_{\text {res }}$ and $Q$ measurement.

Fig. 6: Block diagram of the ring-down readout.

Fig. 7: Circuit diagram of the OTA.

Fig. 8: Circuit diagram of the comparator.

Fig. 9: Circuit diagram of sensor driver.

Fig. 10: Implementation details and timing diagram.

Fig. 11: Effect of parasitic capacitance $C_{p}$ on the TIA's output.

Fig. 12: Micrographs of the readout chip and prototype resonators.

Fig. 13: Measured resolution of $f_{\text {res }}$ as a function $N_{\text {total }}$.

Fig. 14: Measured frequency and quality-factor shift associated with different pressure levels.

Fig. 15: Measured resonance frequency tracking during temperature transients.

\section{Table captions:}

Table I: Performance summary and benchmarking. 


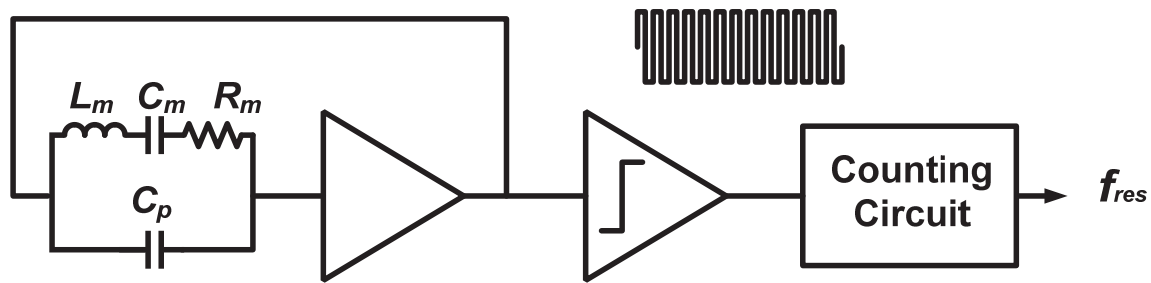

\section{Resonant Sustaining Zero-crossing Sensor Amplifier Detector}

(a)

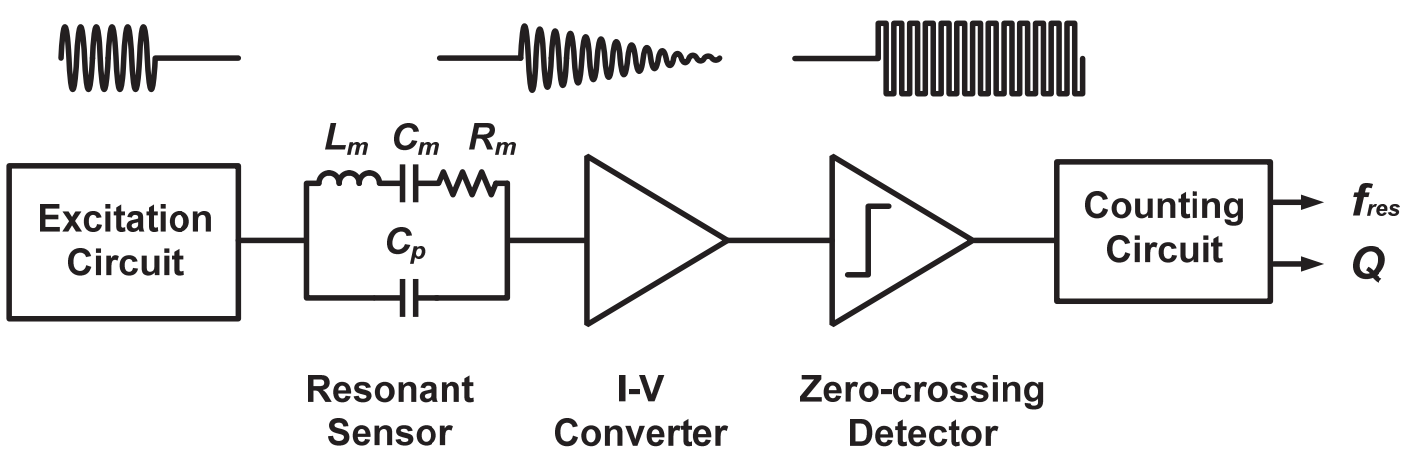

(b)

Fig. 1. Block diagram of (a) an oscillator-based readout circuit; (b) the proposed ring-downbased readout circuit. 


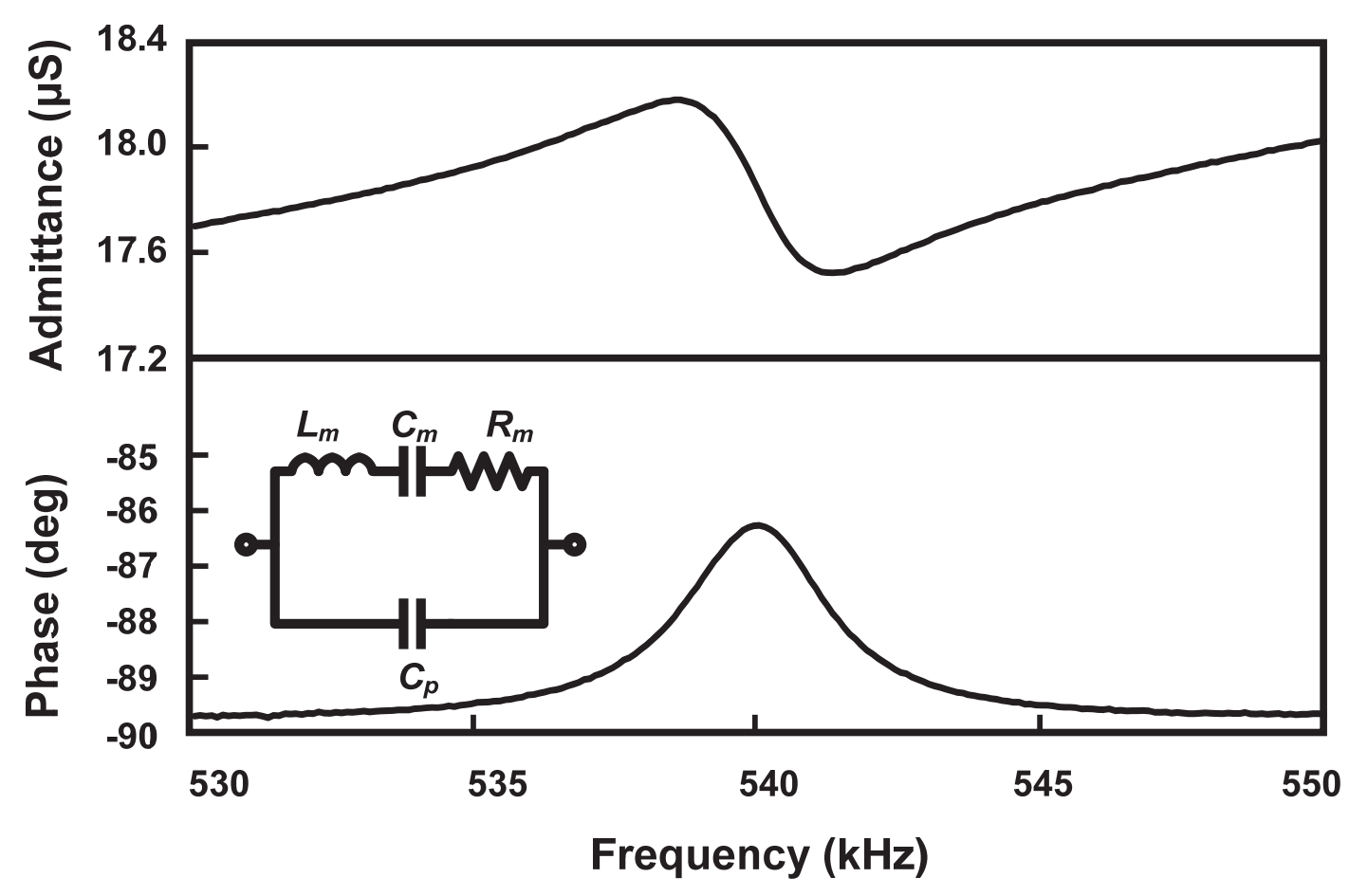

Fig. 2. Measured typical impedance characteristic of the piezo-electrically actuated SiN resonators targeted in this work.

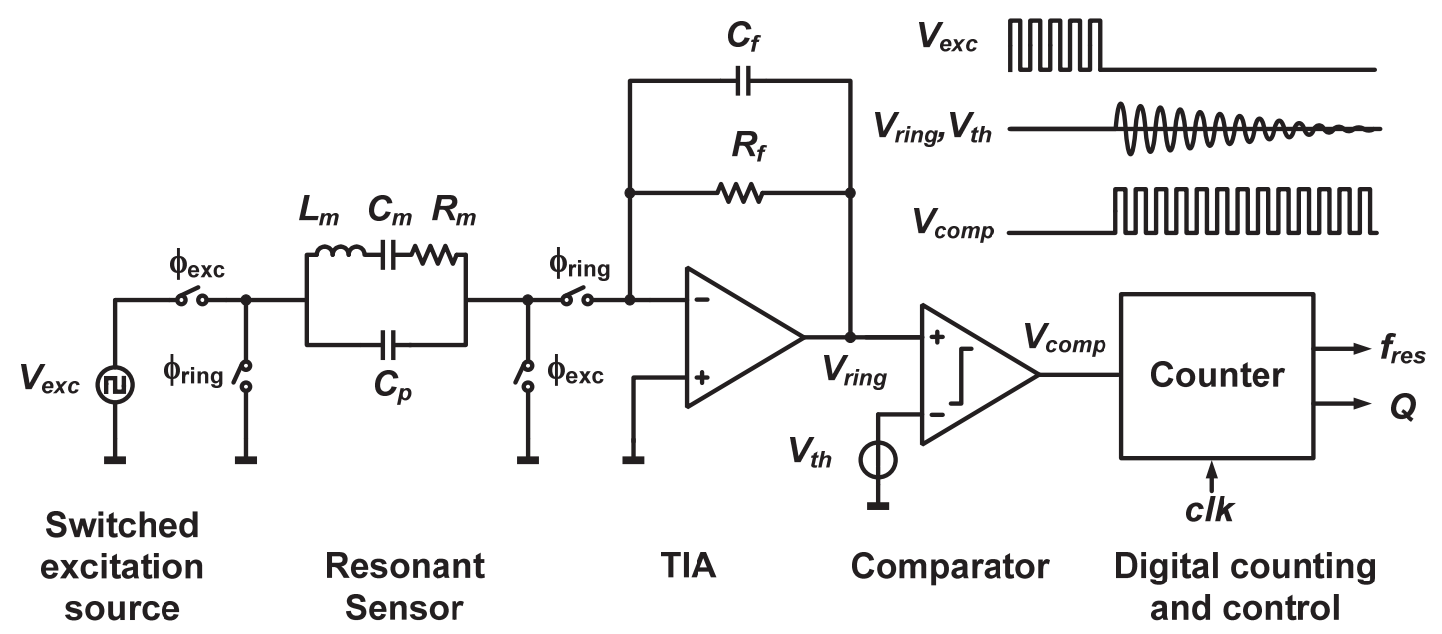

Fig. 3. Basic architecture of the ring-down measurement circuit. 


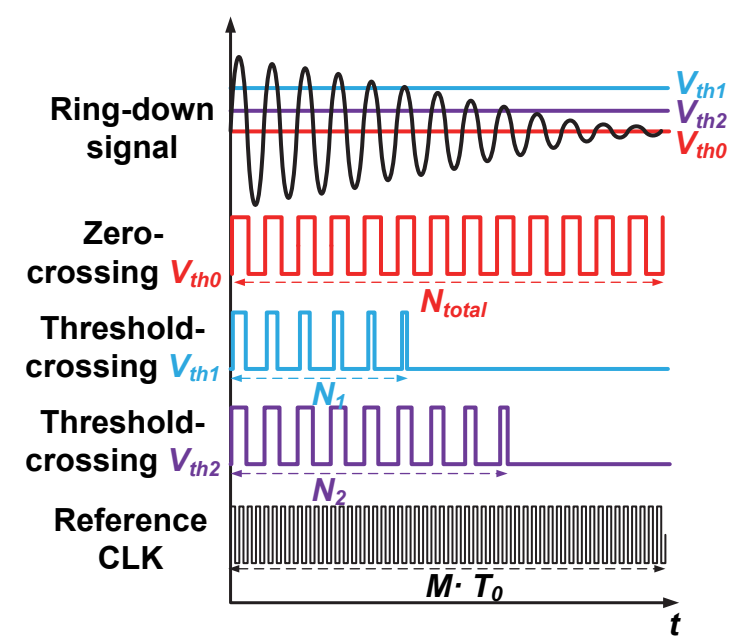

(a)

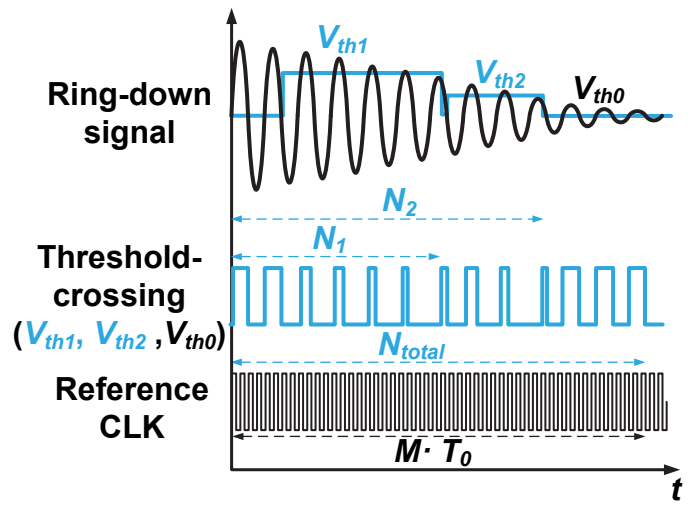

(b)

Fig. 4. Timing diagram of $f_{\text {res }}$ and $Q$ measurements (a) using multiple successive ring-downs;

(b) combined in a single ring-down.

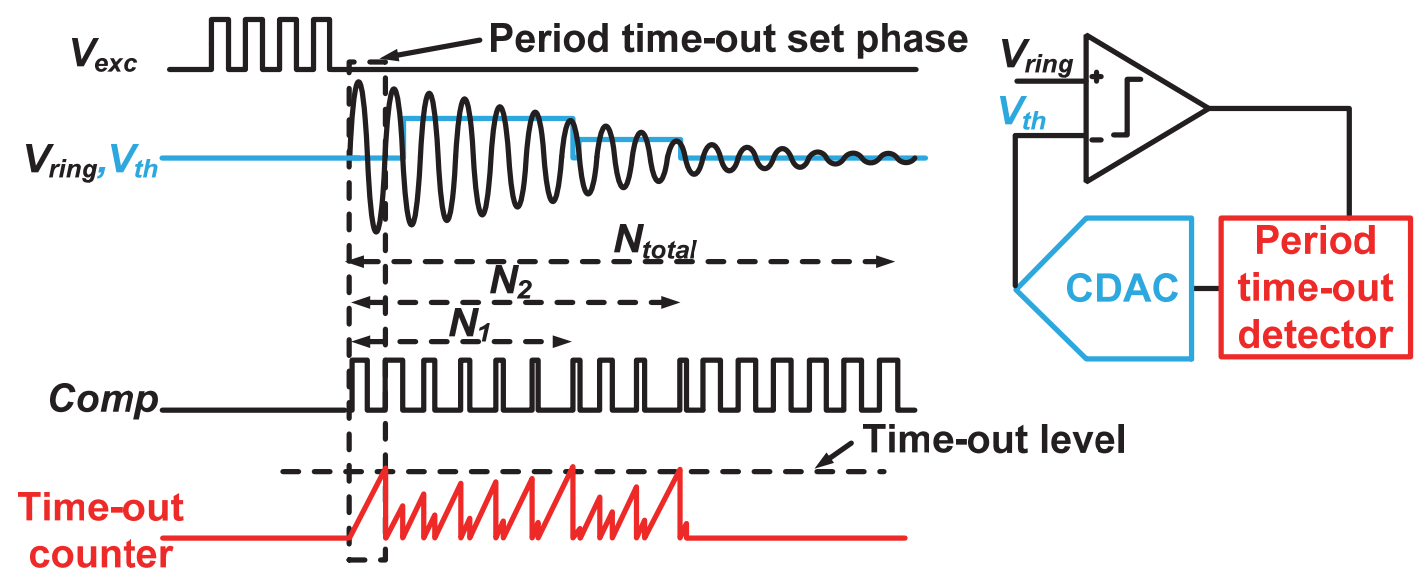

Fig. 5. Dynamic threshold adjustment for the combined $f_{\text {res }}$ and $Q$ measurement. 


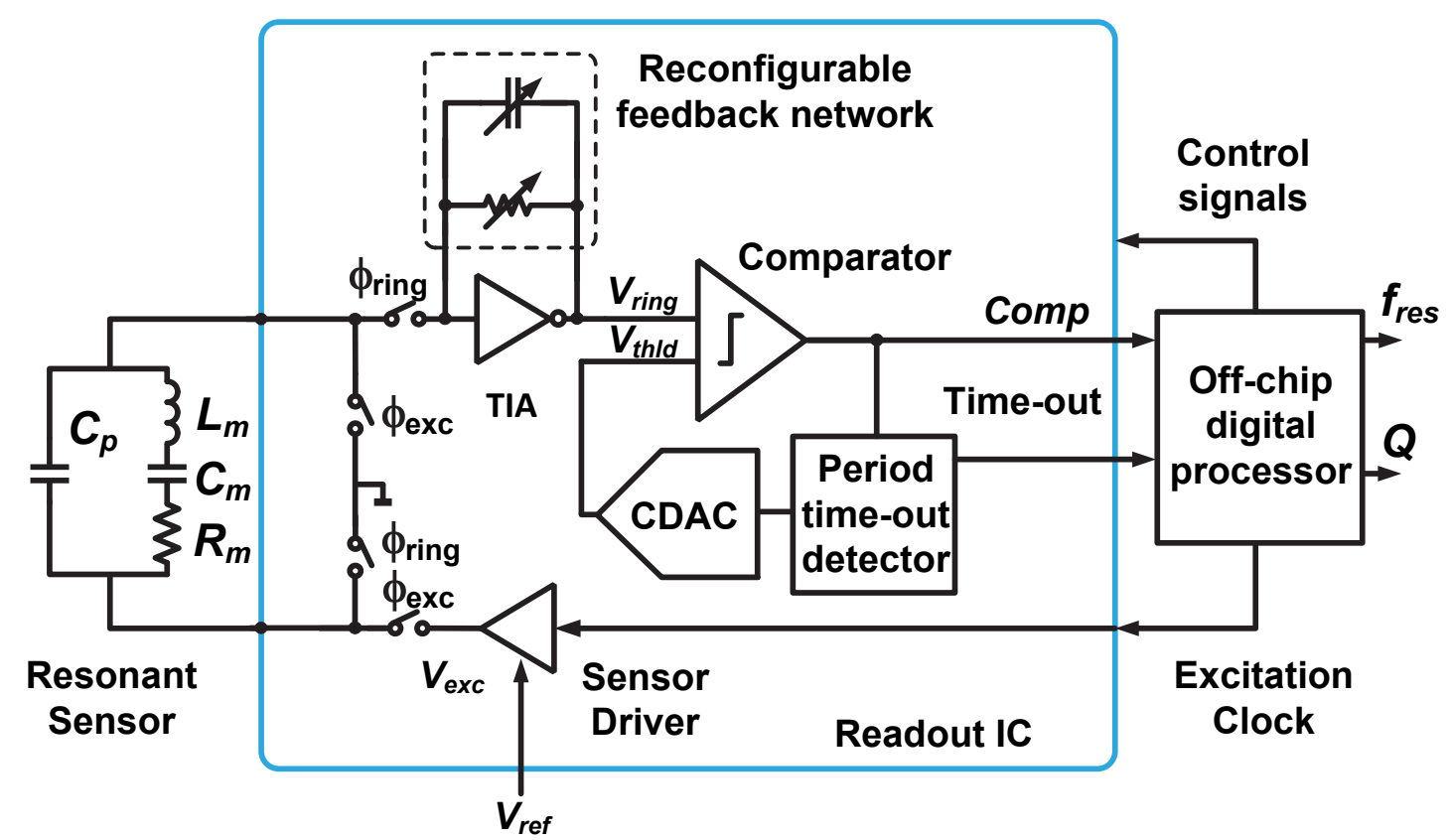

Fig. 6. Block diagram of the ring-down readout.

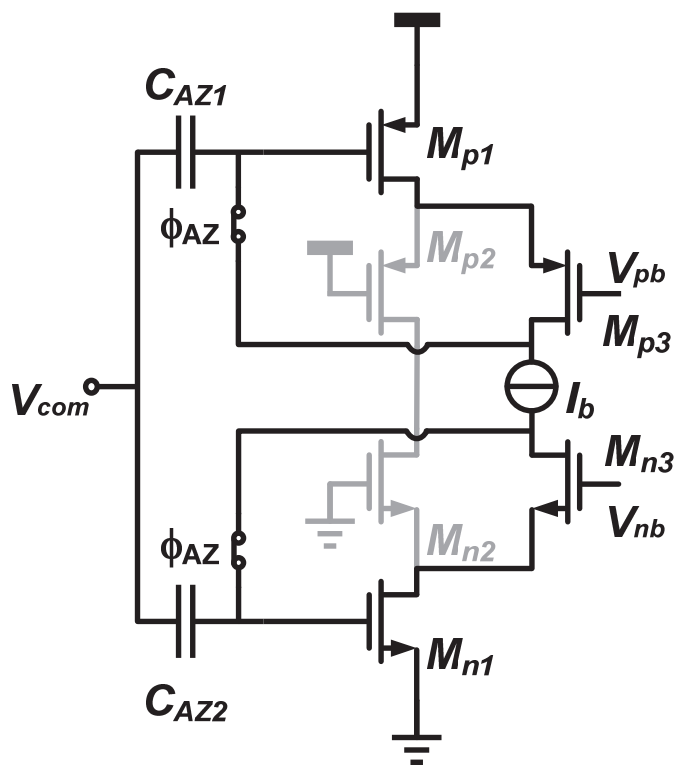

Auto-zeroing Phase

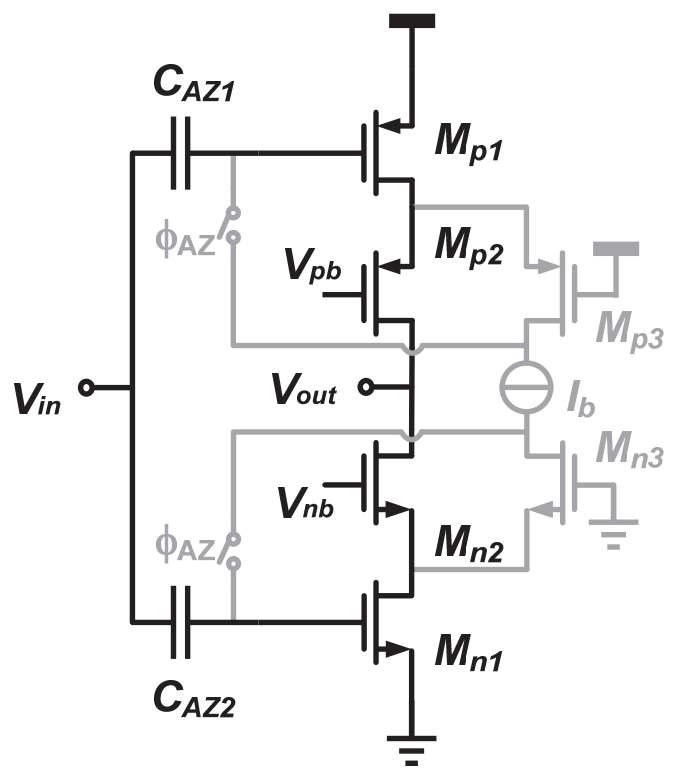

Amplifying Phase

Fig. 7. Circuit diagram of the OTA. 


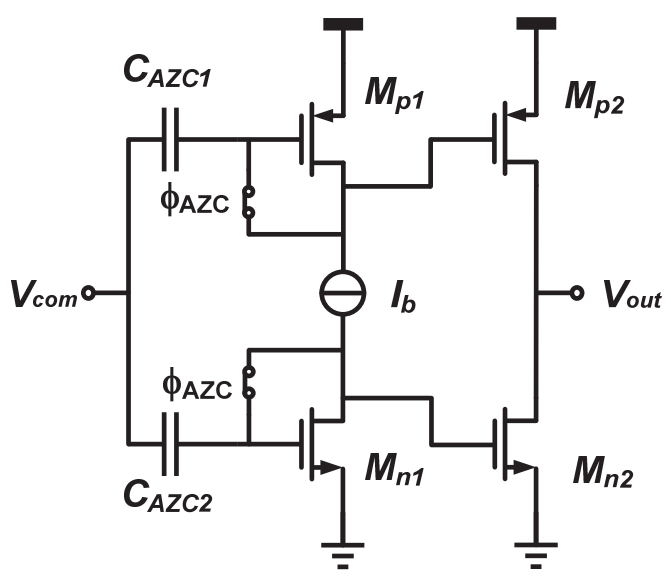

Auto-zeroing Phase

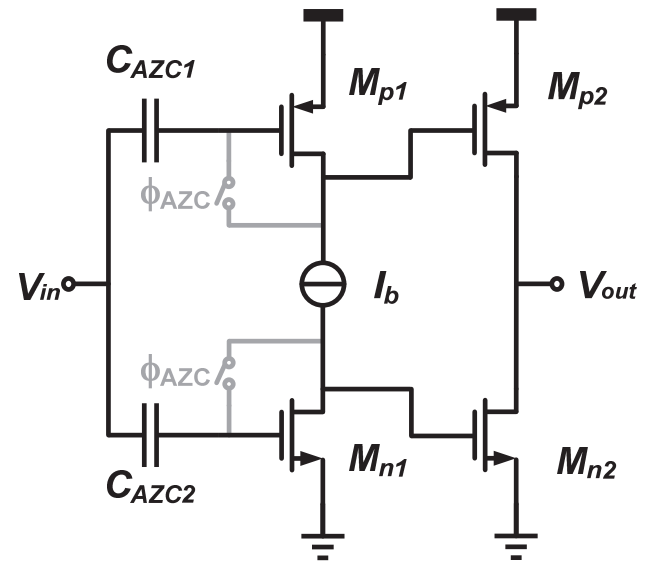

Comparison Phase

Fig. 8. Circuit diagram of the comparator.
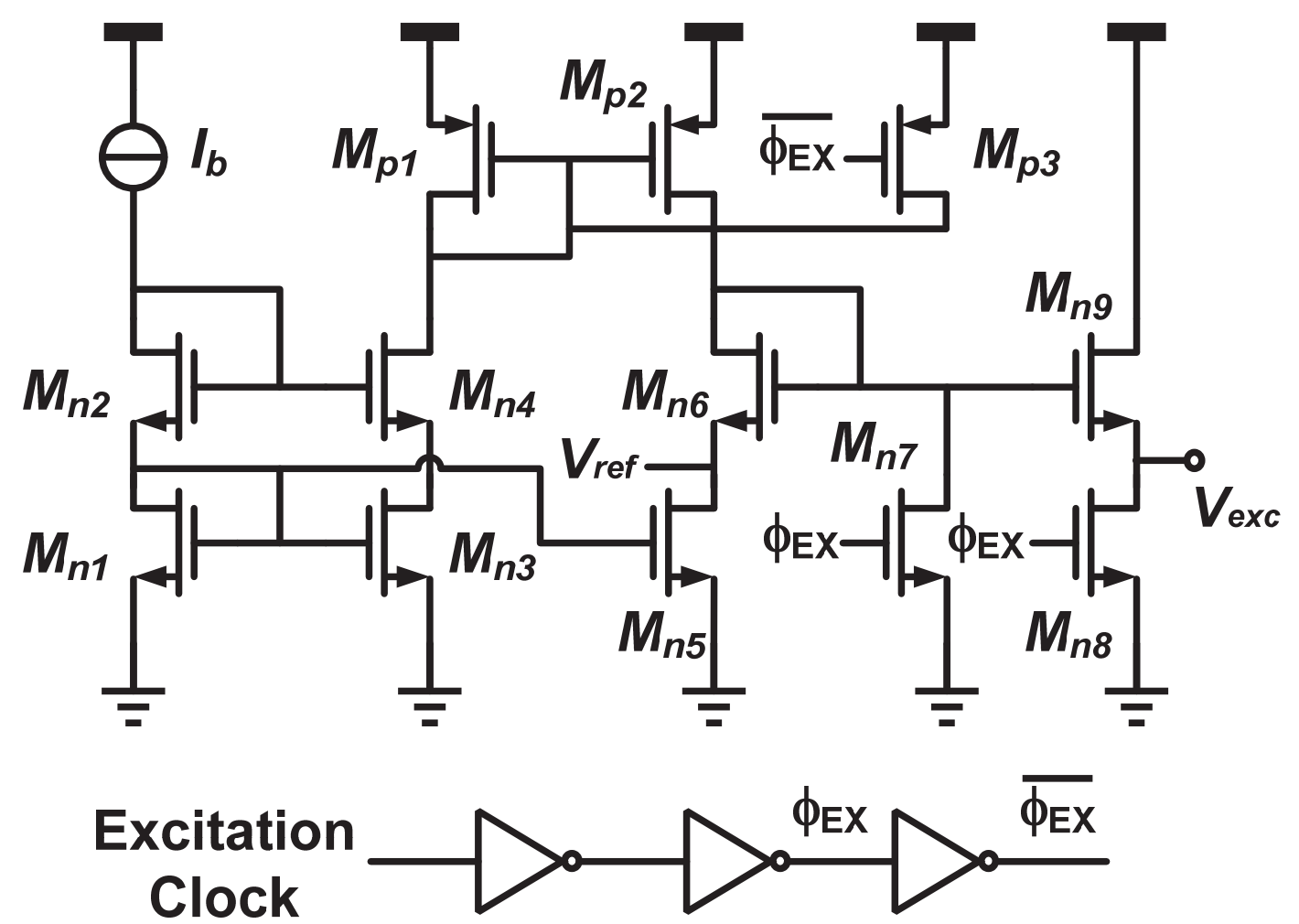

Fig.9. Circuit diagram of the sensor driver. 


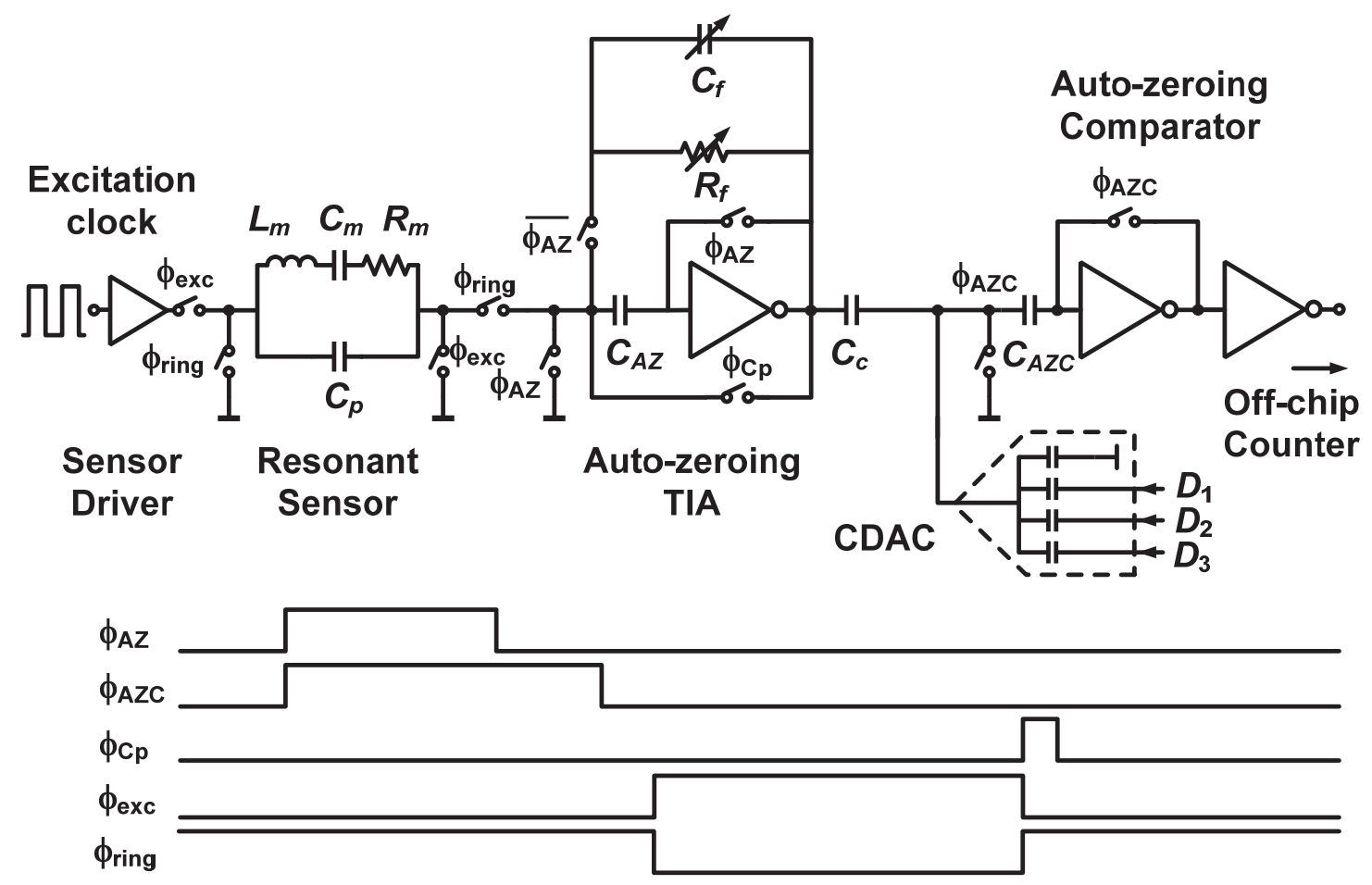

Fig.10. Implementation details and timing diagram.

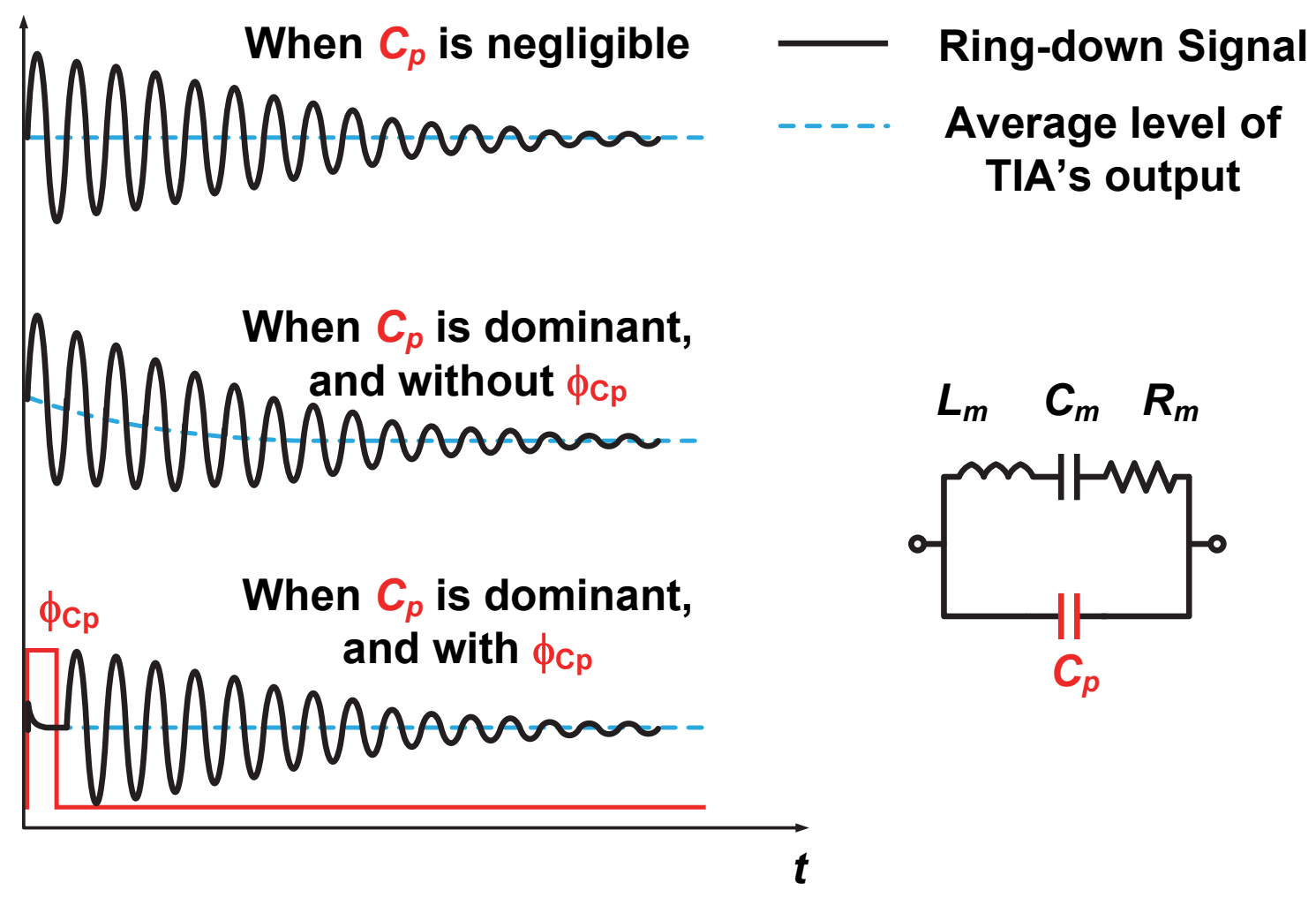

Fig.11. Effect of parasitic capacitance $C_{p}$ on the TIA's output. 


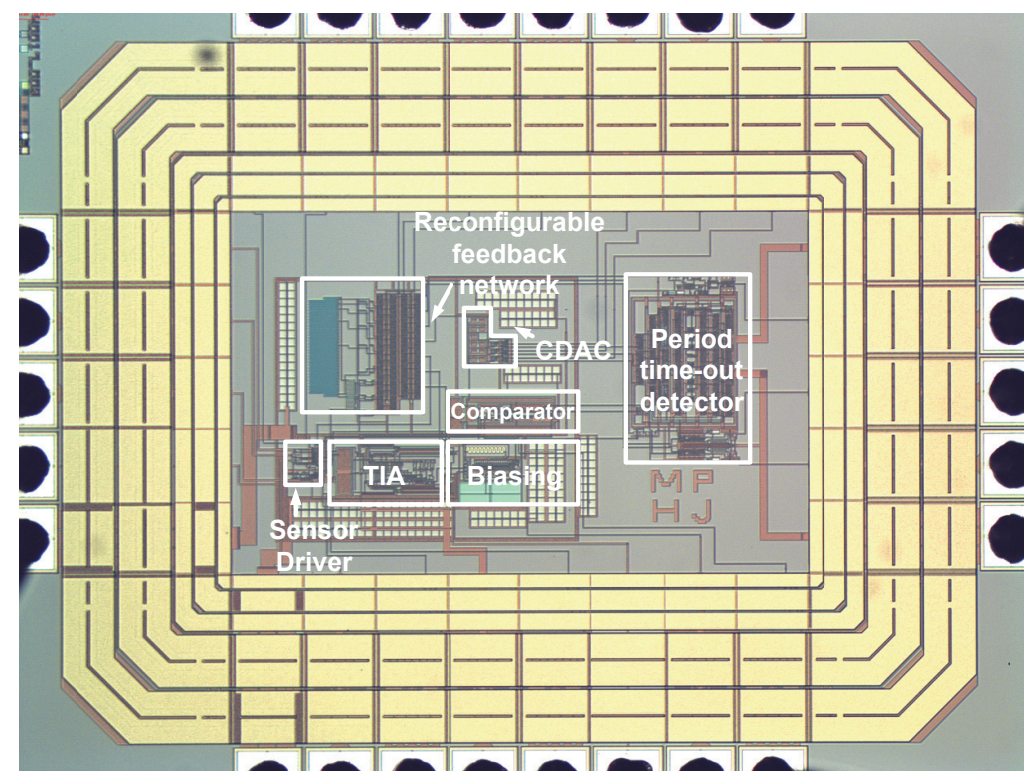

Readout chip

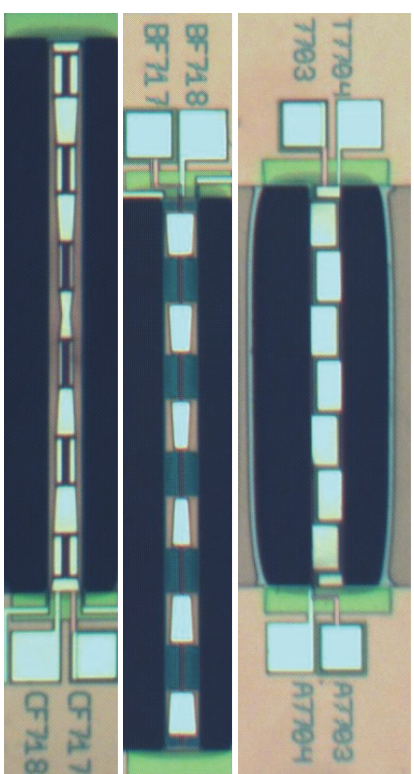

Three Resonators

Fig.12. Micrographs of the readout chip and prototype resonators.

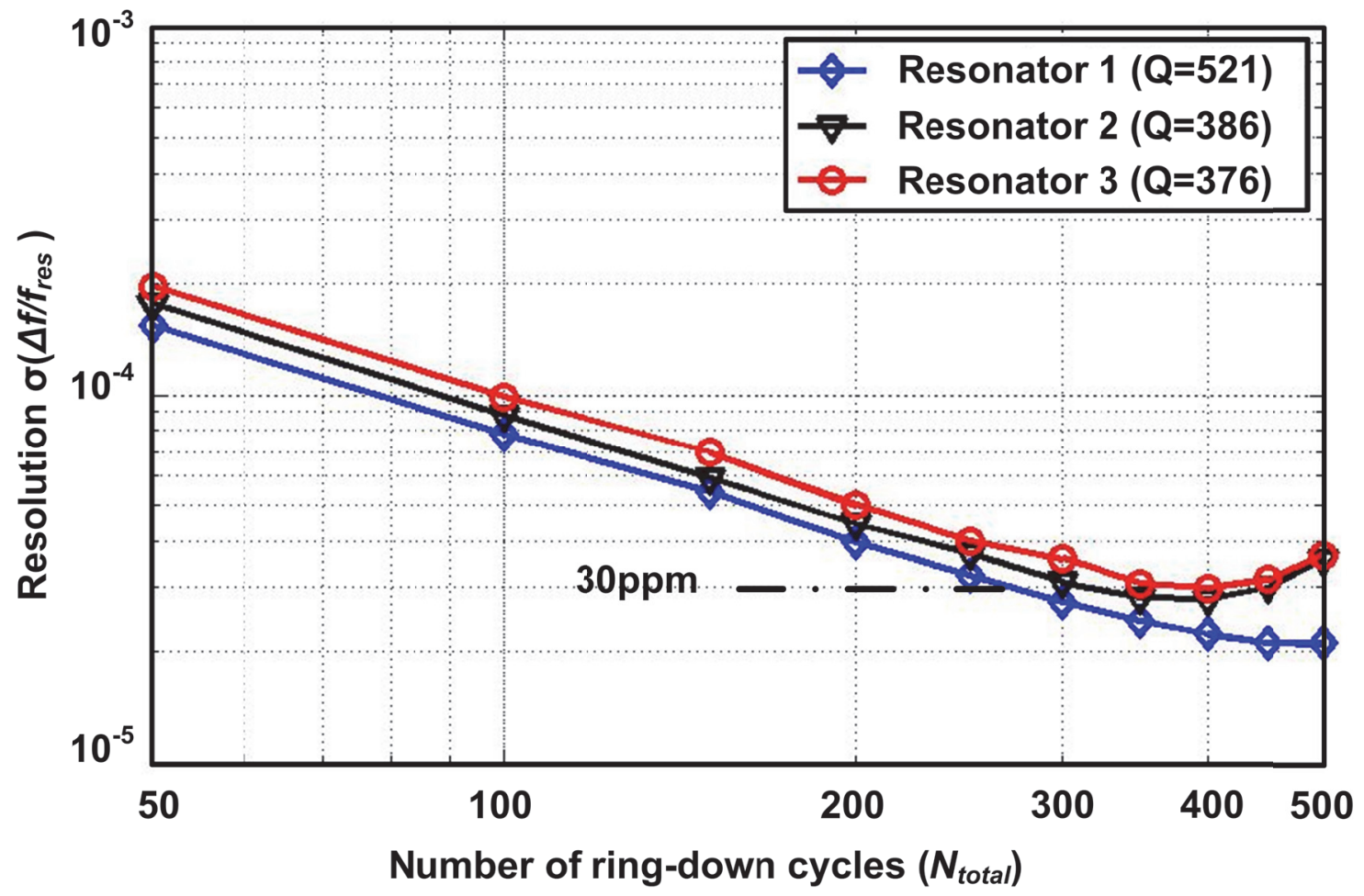

Fig.13. Measured resolution of $f_{\text {res }}$ as a function $N_{\text {total }}$. 


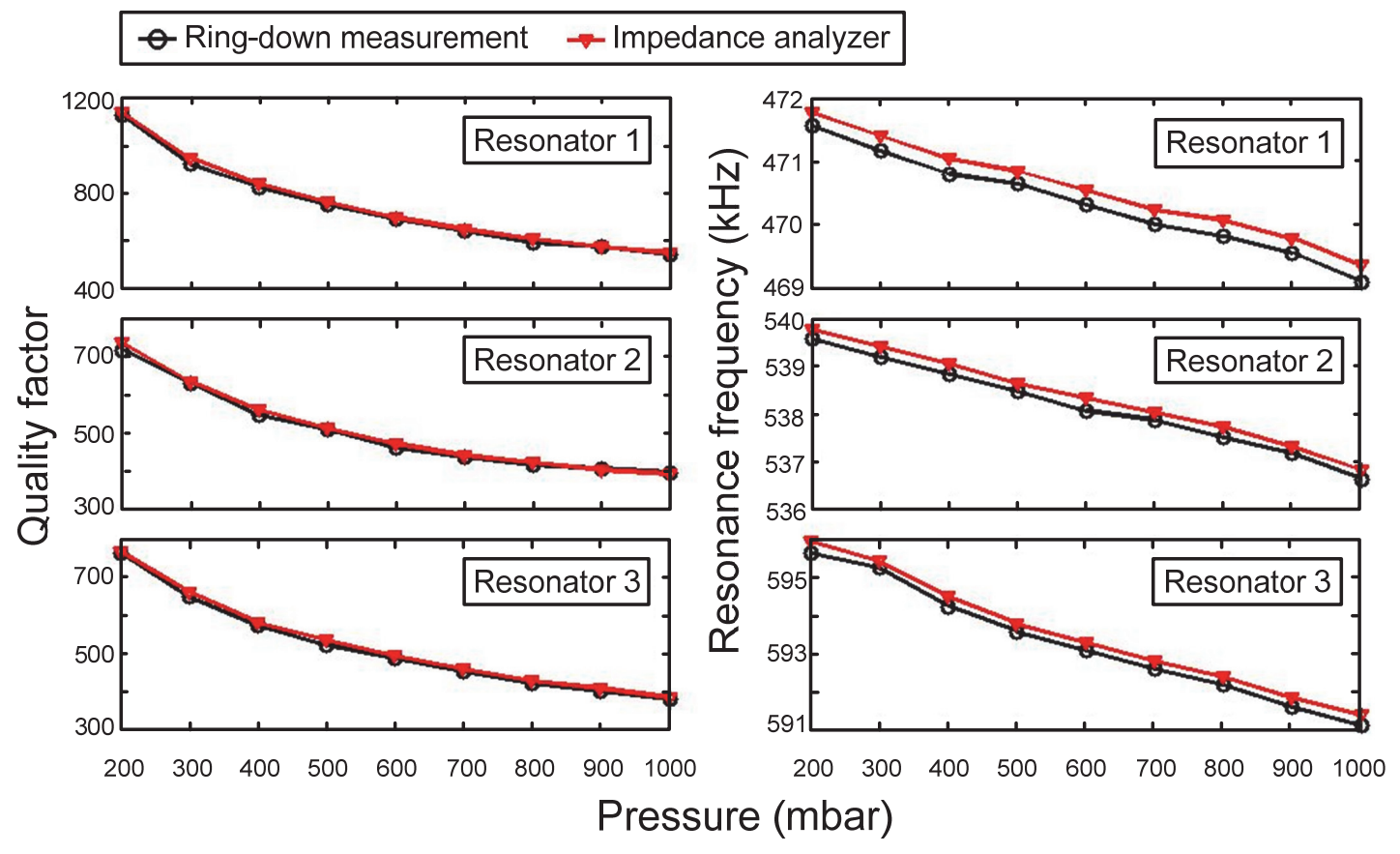

Fig.14. Measured frequency and quality-factor shift associated with different pressure levels.

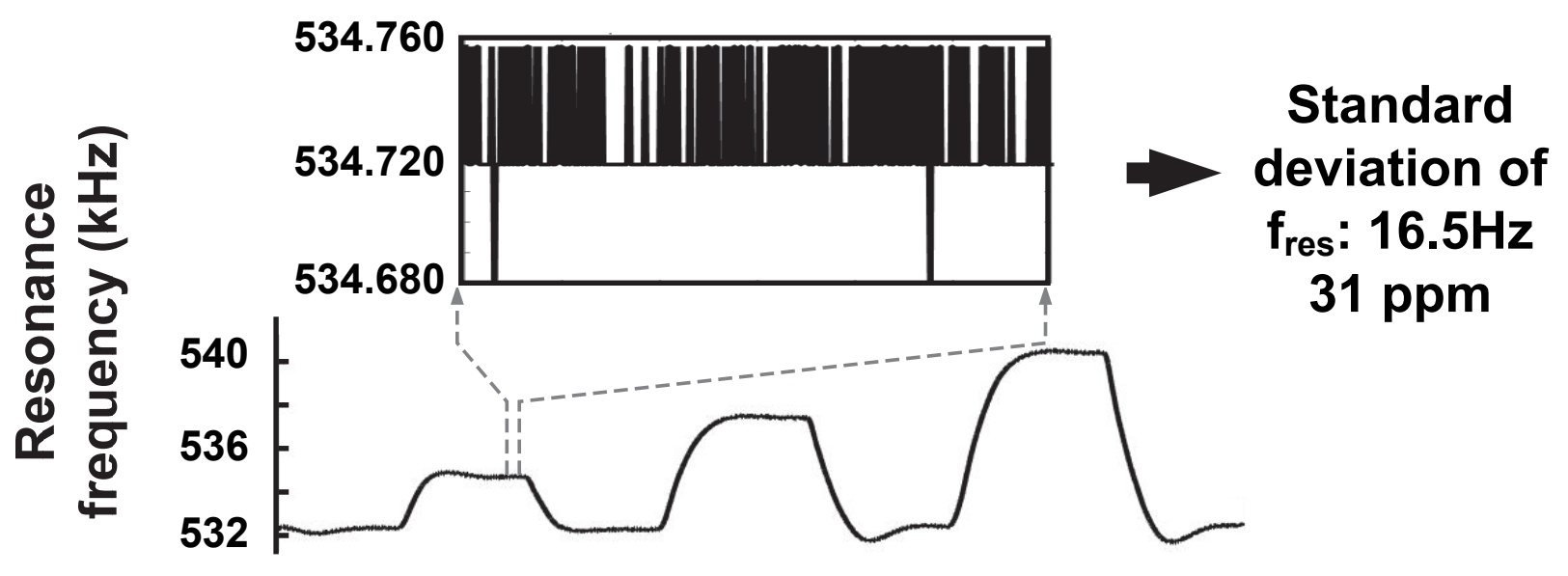

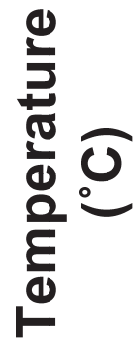

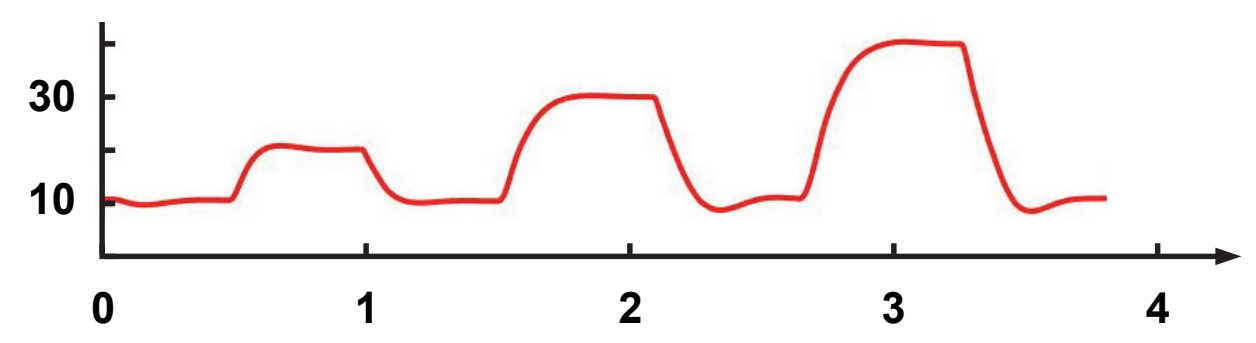

Time (h)

Fig.15. Measured resonance frequency tracking during temperature transients. 
Table I. Performance summary and benchmarking.

\begin{tabular}{|c|c|c|c|c|c|c|c|}
\hline Parameter & $\begin{array}{c}\text { Petrescu } \\
\text { [2] }\end{array}$ & $\begin{array}{c}\text { Pettine } \\
\text { [3] }\end{array}$ & $\begin{array}{c}\text { Zeng } \\
{[8]}\end{array}$ & $\begin{array}{l}\text { Yan } \\
{[9]}\end{array}$ & \multicolumn{3}{|c|}{ This work } \\
\hline Technology & $\begin{array}{l}0.25 \mu \mathrm{m} \\
\mathrm{CMOS}\end{array}$ & $\begin{array}{l}0.25 \mu \mathrm{m} \\
\text { CMOS }\end{array}$ & $\begin{array}{l}0.35 \mu \mathrm{m} \\
\text { CMOS }\end{array}$ & $\begin{array}{l}0.35 \mu \mathrm{m} \\
\text { CMOS }\end{array}$ & \multicolumn{3}{|c|}{$\begin{array}{l}0.35 \mu \mathrm{m} \\
\text { CMOS }\end{array}$} \\
\hline $\begin{array}{c}\text { Supports Q } \\
\text { measurement }\end{array}$ & No & No & Yes & Yes & \multicolumn{3}{|c|}{ Yes } \\
\hline $\begin{array}{c}\text { Supply } \\
\text { voltage }(\mathrm{V})\end{array}$ & 3.3 & 3.3 & 3.3 & 3.3 & \multicolumn{3}{|c|}{1.8} \\
\hline $\begin{array}{c}\text { Supply } \\
\text { current }(\mu \mathrm{A})\end{array}$ & 409 & 409 & 36 & 31.5 & \multicolumn{3}{|c|}{34} \\
\hline $\begin{array}{l}\text { Resonance } \\
\text { freq. }(\mathrm{kHz})\end{array}$ & 1980 & 2170 & 2010 & 535.8 & 469 & 536 & 592 \\
\hline $\begin{array}{l}\text { Quality } \\
\text { factor }\end{array}$ & 280 & 450 & 667 & 386 & 521 & 386 & 376 \\
\hline $\begin{array}{c}\text { Conversion } \\
\text { time (ms) }\end{array}$ & 5000 & 10000 & 3 & 6 & 1.3 & 1.2 & 1.1 \\
\hline $\begin{array}{l}\text { Resolution } \\
\text { (ppm) }\end{array}$ & 27 & 2.4 & 250 & 50 & 21 & 27.7 & 29.8 \\
\hline $\begin{array}{c}\text { Energy } \\
\text { per meas. } \\
(\mu \mathrm{J})\end{array}$ & $6748.5^{1}$ & $13497^{1}$ & $0.356^{2,3}$ & $0.624^{2,3}$ & $0.080^{2}$ & $0.073^{2}$ & $0.067^{2}$ \\
\hline
\end{tabular}

${ }^{1}$ Excludes frequency counter; ${ }^{2}$ excludes counters; ${ }^{3}$ excludes sensor driver 